\title{
Histopathological Changes of the Heart After Neonatal Dexamethasone Treatment: Studies in 4-, 8-, and 50-Week-Old Rats
}

\author{
MIRIAM P. BAL, WILLEM B. DE VRIES, PAUL STEENDIJK, PETRA HOMOET-VAN DER KRAAK, FEIKE R. VAN DER LEIJ, \\ JAN BAAN, MATTHIJS F. M. VAN OOSTERHOUT, AND FRANK VAN BEL
}

\author{
Departments of Neonatology [M.P.B., W.B.V., F.B.], Pathology [P.H.-K., M.F.M.O.], University Medical Center Utrecht, Utrecht 3508 \\ AB, The Netherlands; Department of Cardiology [M.P.B., P.S., J.B.], Leiden University Medical Center, Leiden 2300 RC, \\ The Netherlands; Department of Pediatrics [M.P.B., F.R.L.], University of Groningen, Groningen 9700 RB, The Netherlands; \\ Department of Pathology [M.F.M.O.], St. Antonius Hospital, Nieuwegein 3430 EM, The Netherlands
}

\begin{abstract}
Dexamethasone (Dex), for prevention of chronic lung disease in preterm infants, showed potential negative long-term effects. Studies regarding long-term cardiovascular effects are lacking. We investigated possible histopathological myocardial changes after neonatal Dex in the young and adult rat heart. Rats were treated with Dex on d 1, 2, and $3(0.5,0.3$, and $0.1 \mathrm{mg} / \mathrm{kg})$ of life. Control-pups received saline. At 4, 8, and 50 wk after birth rats were killed and anatomic data collected. Heart tissue was stained with hematoxylin and eosin, Cadherin-periodic acid schiff, and sirius red for cardiomyocyte morphometry and collagen determination. Presence of macrophages and mast cells was analyzed. Cardiomyocyte length of the Dex-treated rats was increased in all three age groups, whereas ventricular weight was reduced. Cardiomyocyte volumes were increased at $50 \mathrm{wk}$ indicating cellular hypertrophy. Collagen content gradually increased with age and was $62 \%$ higher in Dex rats at $50 \mathrm{wk}$. Macrophage focus score and mast cell count were also higher. Neonatal Dex affects normal heart growth resulting in cellular hypertrophy and increased collagen deposition in the adult rat heart. Because previous studies in rats showed premature death, suggesting cardiac failure, cardiovascular follow-up of preterm infants treated with glucocorticoids should be considered. (Pediatr Res 66: 74-79, 2009)
\end{abstract}

$\mathrm{I}_{\mathrm{c}}^{\mathrm{n}}$ preterm infants with severe respiratory distress syndrome, chronic lung disease is a serious complication (1). In the pathogenesis of chronic lung disease an underlying excessive proinflammatory process seems to be involved (2). Glucocorticoids (GCs), in particular dexamethasone (Dex), are widely used to treat or prevent chronic lung disease in preterm infants because of their antiinflammatory action. Moreover, GCs stimulate lung maturation and enhance surfactant production (3).

However, concerns have been raised about the wide range of side effects of GCs (4). These included findings of abnormal brain development in newborn animals treated with systemic steroids (5). Recent reports on follow-up in ex-preterm

Received June 25, 2008; accepted February 2, 2009.

Correspondence: Willem B. de Vries, M.D., Ph.D., Department of Neonatology, KE.04.123.1, University Medical Center Utrecht, The Netherlands; e-mail: w.b.devries-3@umcutrecht.nl

Current address [M.F.M.V.O.]: Department of Pathology [M.F.M.O.], St. Antonius Hospital, Nieuwegein 3430 EM, The Netherlands.

Current address [F.R.V.D.L.]: Unit Life Sciences, Van Hall University of Applied Sciences, PO Box 1528, 8901 BV Leeuwarden, The Netherlands.

Supported by a grant from the Netherlands Heart Foundation (NHS 2001B081) (M.P.B.) and by a personal grant from the Netherlands Heart Foundation (NHS 2004T31) (M.F.M.V.O)

M.P.B. and W.B.V. contributed equally to this work. children neonatally treated with Dex confirm its adverse effects on brain growth (6) and neuromotor developmental outcome (7). With regard to the cardiovascular system, shortterm side-effects, such as myocardial hypertrophy, manifested by increased ventricular septal and left ventricular wall thickness, and hypertension have been reported in animal and human studies $(8-10)$. Recent studies from our group suggested that neonatal Dex treatment may have detrimental long-term effects on the heart, possibly initiated by temporary suppression of the proliferative capacity of cardiomyocytes during and early after treatment (11). These changes may lead to the observed reduction in life expectancy (12).

We therefore performed histopathological and immunohistochemical studies on hearts of rats killed 4, 8, and $50 \mathrm{wk}$ after neonatal Dex treatment and compared the findings with a vehicle treated group of rats to delineate the cardiac effects in the prepubertal and postpubertal and middle-aged period. In this study, we show increased cellular hypertrophy and collagen deposition in the Dex-treated rats of all three age groups, being most pronounced in the 50 -wk-old rats.

\section{MATERIALS AND METHODS}

Animals. The study protocol was approved by the Animal Research Committee of the University of Leiden. The investigation conforms to the Guide for the Care and Use of Laboratory Animals (National Institutes of Health Publication No. 85-23, revised 1996). Pregnant Wistar rats (270-300 g) were housed individually and kept under conventional housing conditions. Pups were born on d 21-22 of gestation. On the day of birth, male pups were selected and randomly divided into treatment and control groups. Only male pups were used to exclude possible influence of gender.

Treatment and control animals were kept separately and placed with foster mothers in groups of 4-6 pups. Rat pups in the treatment-group were injected intraperitoneally with Dex using a 3-d tapering dose following a protocol as used before (13). Consequently, the treated animals received $0.5,0.3$, and 0.1 $\mathrm{mg} / \mathrm{kg}$ bodyweight (Bw) Dex on day 1, 2, and 3 of life, respectively. The control animals received equal volumes $(10 \mu \mathrm{L} / \mathrm{g} \mathrm{Bw})$ sterile pyrogen-free saline (Sal). Temperature and humidity were kept constant and the rats had free access to food and water. An artificial $12 \mathrm{~h}$-light/12 h-dark cycle was used. The rats were weaned on d 21 and studied at 4,8 , or $50 \mathrm{wk}$ of age. Before being killed for the current histopathological study, hemodynamic measurements were performed that were reported elsewhere $(14,15)$. All groups at the various ages (4-, 8-, and 50-wk-old) consisted of eight rats.

The animals were sedated by inhalation of a mixture of halothane (4\%) and oxygen, subsequently general anesthesia was initiated by i.p. injection of a fentanyl-fluanison-midazolam mixture.

Abbreviations: Bw, bodyweight; CSA, cross-sectional area; Dex, dexamethasone; GC(s), glucocorticoid(s); Vw, ventricular weight 


\section{Instrumentation and Histopathological Preparation}

Under general anesthesia, a midsternal thoracotomy was performed and the abdomen of the rats was opened with a medial incision. A $20 \mathrm{G}$ cannula was inserted retrogradely into the abdominal aorta to allow external perfusion of the heart. The hearts were arrested in diastole by slowly infusing $1 \mathrm{~mL} 0.1$ $\mathrm{M}$ cadmiumchloride, opening the right atrium to allow drainage. Coronary vasodilatation was achieved by 3 min of perfusion with a mixture of $\mathrm{NaCl}$ and nitroprusside $(0.1 \mathrm{mg} / \mathrm{mL}$ using a reservoir at $\sim 70 \mathrm{~cm}$ height $)$ followed by 3 min formalin solution (2\%) for perfusion fixation. After excision, the hearts were immersion fixed in phosphate-buffered formalin $4 \%$ for at least $48 \mathrm{~h}$. Remaining extra-cardiac structures and the atria were removed from the hearts and total ventricular weight $(\mathrm{Vw})$ was determined. The ventricles were cut in coronal sections of $\sim 2$-mm thickness and embedded in paraffin. Subsequently, the hearts were sectioned parallel to the equator in $3 \mu \mathrm{m}$ slices.

Hematoxylin and eosin $(\boldsymbol{H} \& \boldsymbol{E})$. H\&E staining was performed for general histopathological assessment. Subsequently an H\&E-stained slice at the level of the papillary muscles was selected for measurement of left ventricular free wall thickness.

Sirius red. Sirius red staining (Polysciences, Warrington, PA) was performed in $3-\mu \mathrm{m}$ sections after pretreatment with picrinic acid to determine collagen content. The collagen-positive area was quantified using ImagePro analysis software (Media Cybernetics, Inc, Bethesda, MD) in a total of 40 fields of four predefined regions (left ventricular free wall, anterior wall, posterior wall, and interventricular septum) using a final magnification of $\times 200$. Collagen content was expressed as fraction of the total myocardial cross-sectional area.

Cadherin-periodic acid schiff (PAS). Cadherin-PAS staining, modified from Bruel and Nyengaard (16) was used for the morphometric measurements of the cardiomyocytes. The Cadherin-PAS provides staining of the intercalated discs and the lateral sarcolemma. First, $3-\mu \mathrm{m}$ sections of the ventricles were deparaffinized. After EDTA boiling, the slides were incubated with Cadherin (pan, C1821, Sigma-Aldrich Chemical Co., Denmark) for $1 \mathrm{~h}$ and then with RAMPO (P0161, Dakocytomation, Denmark) for $30 \mathrm{~min}$. Subsequently, a Horse Radish-labeled antibody (powervision poly HRP-anti-Rabbit IgG, immunologic) was used. After developing the slides with diaminobenzidene acid and washing, the PAS staining was performed. The slides were incubated with $1 \%$ periodic acid for $10 \mathrm{~min}$, followed by incubation with Schiff's reagent (Merck, Whitehouse Station, NJ) for $30 \mathrm{~min}$. Finally, the slides were counter-stained with hematoxylin.

Macrophage staining. Macrophage staining (ED-1, Acris antibodies, homologue of human CD68) was performed only on the 50-wk-old rats to quantify the monocytic infiltrates. The deparaffinized slides were citrate boiled, incubated with ED1 for $1 \mathrm{~h}$, and incubated with RAMPO followed by a Swar-PO amplification with Nova red as chromogen.

Mast cell staining. Mast cell staining [Bismarck brown (Bdh, Chroma, Münster, Germany) for $90 \mathrm{~min}$ ] was performed on the 50 -wk-old rat hearts to ensure that CD68 positive cells were of macrophage origin and not mast cells.

Cardiomyocyte morphometry. Morphometry was performed as described by van Oosterhout et al. (17) using the Cadherin-PAS staining. In brief, myocytes from predefined myocardial areas (see later) were visualized with a microscope (Zeiss Axiomat, Oberkochen, Germany; final magnification $400 \times$ ) equipped with a digital camera (Nikon Eclipse E800, Tokyo, Japan) and coupled to a personal computer equipped with dedicated software (QProdit, Leica Microsystems, Wetzlar, Germany). Myocyte cross-sectional area (CSA) was determined in the left ventricular free wall and the interventricular septum from both subepicardium and subendocardium by tracing the boundaries of the cells, using only those cells in witch a centrally located nucleus was visible. For the subepicardium of the interventricular septum, the subendocardial located myocytes of the right ventricle were used. From each site, 25 myocytes were used for determination of CSA, rendering 100 cell measurements. In the middle third of the wall thickness of the left ventricular free wall and the interventricular septum, longitudinally oriented myocytes (40 from each site, rendering 80 cell measurements for each animal) were selected and the cell boundaries were traced. Diameter and longitudinal sectional area of these cells were automatically determined by dedicated software (QProdit, Leica Microsystems). Only those myocytes in which the nucleus was centrally located within the cell and with intercalated discs visible at both ends of the cell were used to ensure that the long axis of the myocyte was perpendicular to the microscope objective (18). Effective cardiomyocyte length was defined in this study as longitudinal sectional area divided by the diameter of the longitudinally oriented cells. Cardiomyocyte volume was calculated as longitudinal sectional area multiplied by diameter $\times \pi / 4$ on the assumption of a cylindrical configuration. Except for the data reported in Table 1 (see later), the CSA value for each heart was determined as the median from the subepicardial and subendocardial cardiomyocytes, respectively. From the longitudinally oriented cardiomyocytes, the median of the volume, length, and width was calculated for each heart and used for statistical analysis.

Macrophage focus score and mast cell count. In the 50-wk-old rats of both groups, the number of macrophage foci was quantified. The observer was blinded to the treatment protocol. In one section, comprising the cross section of the whole left ventricle at the level of the papillary muscles, the number of macrophage foci was counted. This number was related to the total area of the left ventricular cross section and expressed as number of foci/10 $\mathrm{mm}^{2}$. A focus was defined as at least five adjacent ED1 positive macrophages. Because ED-1 may also stain mast cells, a parallel section was also stained with the mast cell specific Bismarck Brown staining. Positive cells were enumerated using a $\times 40$ objective lens. Twenty randomly chosen fields were analyzed and counted (comprising $\sim 80 \%$ of the cross section of the left ventricle).

Statistics. Data are presented as mean \pm SD. Anatomical data of the age groups were compared using unpaired $t$ tests. Morphometric data and collagen content for each age group were analyzed using Mann-Whitney $U$ tests because of a nonlinear distribution of the data. The $p$-values of $<0.05$ were considered statistically significant.

\section{RESULTS}

Anatomical Parameters. Anatomical data for Dex-treated and Sal-treated rats are summarized in Table 2. In the 4-wkold rats, $\mathrm{Bw}, \mathrm{Vw}$, and the ratio of $\mathrm{Vw} / \mathrm{Bw}$ were lower in the Dex-treated rats by, respectively, $16 \%(p<0.001), 22 \%(p<$ $0.001)$, and $8 \%(p<0.05)$. In the 8 -wk-old rats, Vw was $11 \%$ lower in Dex $(p<0.05)$, but no difference was found for Bw or $\mathrm{Vw} / \mathrm{Bw}$. No differences between Dex and Sal were found for $\mathrm{Bw}, \mathrm{Vw}$, or ratio $\mathrm{Vw} / \mathrm{Bw}$ in the 50 -wk-old rats. Ventricular wall thickness was lower by $20 \%(p<0.01)$ in the Dextreated rats at $4 \mathrm{wk}$, no differences were found in the 8- and 50-wk-old rats.

Cardiomyocyte morphometry. At $4 \mathrm{wk}$, there were no differences in mean cardiomyocyte CSA between Sal- and Dex-treated animals. However, as shown in Figure 1, at 8 and 50 wk mean CSA was increased by $13 \%(p<0.01)$ and $30 \%$ $(p<0.01)$, respectively, in Dex-treated rats compared with Sal-treated rats. The frequency distribution of CSA showed a clear shift toward increased CSA in the Dex-treated rats compared with the Sal-treated control rats.

Because it is well known that cardiomyocyte size is not equally distributed transmurally (19-21), we analyzed the CSA of subepicardially and subendocardially located cardio-

Table 1. Cross-sectional area of cardiomyocytes (mean $\pm S D$ ) in 4-, 8-, and 50-wk-old rats neonatally treated with dexamethasone or saline

\begin{tabular}{|c|c|c|c|c|c|c|c|c|c|}
\hline & \multicolumn{3}{|c|}{ 4-wk-old rats } & \multicolumn{3}{|c|}{ 8-wk-old rats } & \multicolumn{3}{|c|}{ 50-wk-old rats } \\
\hline & Sal & Dex & $\begin{array}{c}p \\
\text { (Dex vs. Sal) }\end{array}$ & Sal & Dex & $\begin{array}{c}p \\
\text { (Dex vs. Sal) }\end{array}$ & Sal & Dex & $\begin{array}{c}p \\
\text { (Dex } v s . \text { Sal) }\end{array}$ \\
\hline Subendocardial $\left(\mu \mathrm{m}^{2}\right)$ & $207.5 \pm 38.6$ & $225.5 \pm 56.1$ & ns & $358.8 \pm 38.3$ & $448.3 \pm 51.2$ & $<0.005$ & $520.8 \pm 93.0$ & $723.4 \pm 102.0$ & $<0.001$ \\
\hline Subepicardial $\left(\mu \mathrm{m}^{2}\right)$ & $166.2 \pm 23.3$ & $162.3 \pm 40.1$ & ns & $328.7 \pm 29.0$ & $338.9 \pm 35.6$ & $\mathrm{~ns}$ & $463.1 \pm 53.7$ & $631.1 \pm 107.6$ & $<0.005$ \\
\hline $\begin{array}{l}p \text {, subendocardial } v s . \\
\text { subepicardial }\end{array}$ & $<0.05$ & $<0.05$ & & ns & $<0.0005$ & & $\mathrm{~ns}$ & $\mathrm{~ns}$ & \\
\hline
\end{tabular}

ns, not significant. 
Table 2. Anatomical parameters of the heart (mean $\pm S D)$ in 4-, 8-, and 50-wk-old rats neonatally treated with dexamethasone or saline

\begin{tabular}{|c|c|c|c|c|c|c|c|c|c|}
\hline & \multicolumn{3}{|c|}{ 4-wk-old rats } & \multicolumn{3}{|c|}{ 8-wk-old rats } & \multicolumn{3}{|c|}{ 50-wk-old rats } \\
\hline & Sal & Dex & $p$ & Sal & Dex & $p$ & Sal & Dex & $p$ \\
\hline $\mathrm{Bw}(\mathrm{g})$ & $86 \pm 9$ & $72 \pm 3$ & $<0.001$ & $259 \pm 23$ & $243 \pm 8$ & $\mathrm{~ns}$ & $518 \pm 36$ & $515 \pm 32$ & $\mathrm{~ns}$ \\
\hline $\mathrm{Vw}(\mathrm{g})$ & $0.36 \pm 0.02$ & $0.28 \pm 0.03$ & $<0.001$ & $0.95 \pm 0.09$ & $0.84 \pm 0.07$ & $<0.05$ & $1.32 \pm 0.13$ & $1.23 \pm 0.10$ & $\mathrm{~ns}$ \\
\hline $\mathrm{Vw} / \mathrm{Bw}(\mathrm{g} / \mathrm{kg})$ & $4.2 \pm 0.3$ & $3.8 \pm 0.34$ & $<0.05$ & $3.7 \pm 0.2$ & $3.5 \pm 0.36$ & $\mathrm{~ns}$ & $2.6 \pm 0.32$ & $2.4 \pm 0.16$ & $\mathrm{~ns}$ \\
\hline WT (mm) & $1.47 \pm 0.19$ & $1.18 \pm 0.18$ & $<0.01$ & $2.45 \pm 0.14$ & $2.34 \pm 0.10$ & ns & $2.74 \pm 0.25$ & $2.63 \pm 0.23$ & $\mathrm{~ns}$ \\
\hline \multicolumn{10}{|l|}{ Cardiomyocyte dimensions } \\
\hline Length $(\mu \mathrm{m})$ & $60.9 \pm 5.5$ & $69.3 \pm 4.1$ & $<0.05$ & $79.7 \pm 6.7$ & $90.9 \pm 6.1$ & $<0.01$ & $93.9 \pm 11.5$ & $111.9 \pm 9.9$ & $<0.05$ \\
\hline Width $(\mu \mathrm{m})$ & $12.0 \pm 0.7$ & $12.1 \pm 0.6$ & $\mathrm{~ns}$ & $15.7 \pm 1.3$ & $15.9 \pm 1.6$ & ns & $17.7 \pm 1.8$ & $20.7 \pm 1.6$ & $<0.01$ \\
\hline Volume $\left(\times 10^{3} \mu \mathrm{m}^{3}\right)$ & $7.1 \pm 0.9$ & $8.1 \pm 1.2$ & $\mathrm{~ns}$ & $15.7 \pm 4.0$ & $18.1 \pm 3.1$ & $\mathrm{~ns}$ & $23.8 \pm 6.0$ & $38.6 \pm 9.0$ & $<0.01$ \\
\hline
\end{tabular}

$p$-value Dex vs. Sal.

WT, wall thickness; ns, not significant.
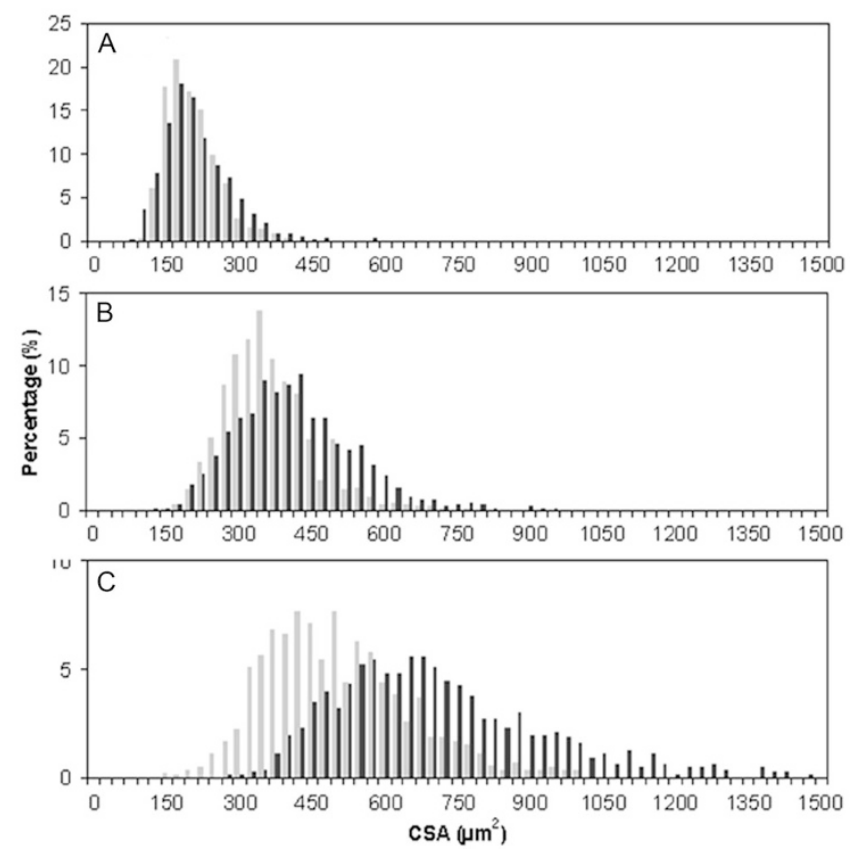

Figure 1. Cross-sectional area of cardiomyocytes in 4-(A), 8-(B), and 50-(C) week-old rats. Histograms reflect individual measurements of all myocytes (100 cells/animal) for all animals; filled bars indicate Dex and open bars indicate Sal.

myocytes separately. In addition, in this subanalysis (Table 1) the CSA is significantly increased at $8 \mathrm{wk}$ (subendocardial), at 50 wk and not at 4 wk in Dex compared with Sal.

Cardiomyocyte width and length were determined in a total of 80 longitudinally oriented cardiomyocytes for each animal. In Figure 2, representative examples of the Cadherin-Pas staining for 50-wk-old Sal- and Dex-treated rats are shown. Longitudinally sectioned myocytes showed an increased distance between intercalated discs in the Dex-treated rats compared with Sal, indicating increased length of the cardiomyocytes.

Figure 3 shows the relation between cardiomyocyte volume and $\mathrm{Vw}$. In the Sal-treated rats a practically linear relation between cell volume and $\mathrm{Vw}_{\mathrm{w}}$ was found. However, in the Dex-treated rats, this relation was nonlinear with a disproportional increase in cell volume between 8 and 50 wk of age, indicating relatively smaller hearts and larger cardiomyocytes. After Dex treatment, cell volume was higher, though not significantly, at 4 and $8 \mathrm{wk}$ by $15 \%$. At $50 \mathrm{wk}$, cell volume was significantly increased by $62 \%(p<0.01)$, respectively.
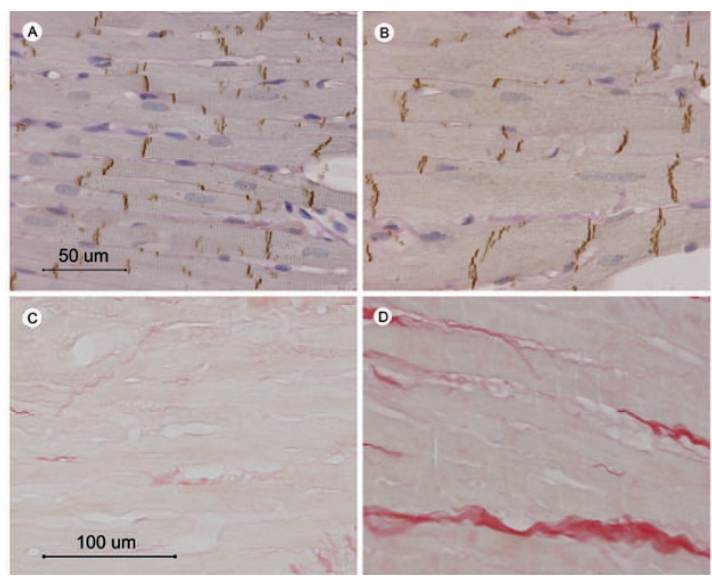

Figure 2. Histology of the hearts of 50-wk-old rats neonatally treated with Dex or saline (Sal). Representative examples of the Cadherin-Pas staining for 50 -wk-old Sal- $(A)$ and Dex-treated $(B)$ rats. Increased collagen content in the 50 -wk-old Dex-treated rat $(D)$ compared with the Sal-treated rat $(C)$.

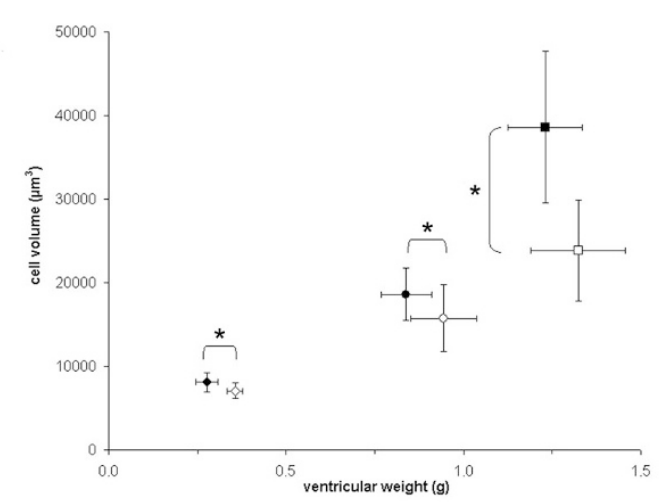

Figure 3. Cardiomyocyte volume vs. Vw. Open symbols, Sal-treated rats; closed symbols, Dex-treated rats. $\diamond=4 \mathrm{wk}, \bigcirc=8 \mathrm{wk}$, and $\square=50 \mathrm{wk}$. $* p<0.05$ Dex vs. Sal.

At all ages this increased cell volume in the Dex-groups was accompanied by a significant increase of the cardiomyocyte long axis $[4 \mathrm{wk},+14 \%(p<0.05) ; 8 \mathrm{wk},+14 \%(p<$ $0.01) ; 50 \mathrm{wk},+13 \%(p<0.05)]$. The width of the cardiomyocytes did not increase in the 4- and 8-wk-old rats, but eventually increased by $20 \%(p<0.01)$ in the 50-wk-old Dex-treated rats. The relation between the myocyte width and length is depicted in Figure 4. It shows that cellular enlargement in the Dex-groups was predominantly due to lengthening 


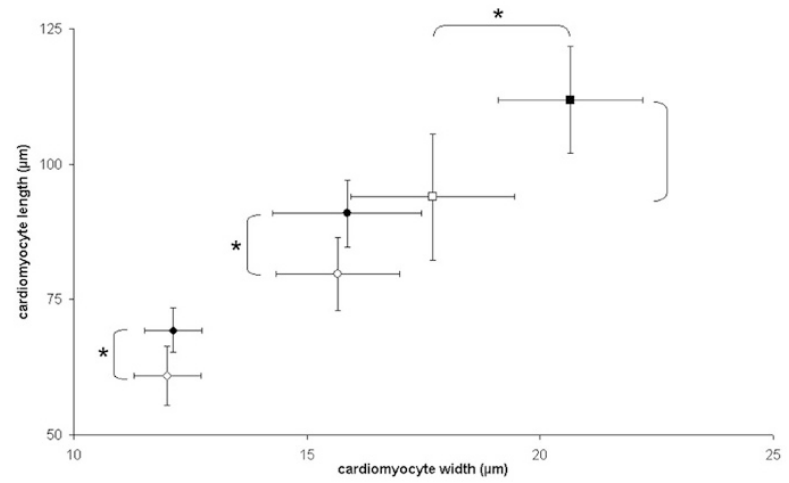

Figure 4. Cardiomyocyte length $v s$. width. Open symbols, Sal-treated rats; closed symbols, Dex-treated rats. $224=4 \mathrm{wk}, \bigcirc=8 \mathrm{wk}$, and $\square=50 \mathrm{wk}$. $* p<0.05$ Dex vs. Sal.

in the 4- and 8-wk-old rats, whereas at 50 wk both length and width were increased compared with Sal. These findings suggest eccentric hypertrophy at the younger ages and concentric hypertrophy in the older animals.

Collagen content measured as a fraction of the total myocardial area tended to be higher already at 4 wk (Dex: $0.74 \pm$ $0.46 \%$ vs. Sal: $0.50 \pm 0.33 \% ; p=0.248)$ and $8 \mathrm{wk}($ Dex: $1.16 \pm 0.70 \%$ vs. Sal: $0.74 \pm 0.51 \% ; p=0.141)$. In the 50 -wk-old Dex-treated rats, the collagen content was significantly (62\%) higher than in control rats [Dex: $2.20 \pm 0.60 \%$ vs. Sal: $1.36 \pm 0.21 \%(p<0.01)]$. Figure 2 (bottom) shows typical examples to illustrate the increased collagen content in the 50-wk-old Dex-treated rats.

Macrophage focus score and mast cell count in 50-wk-old rats. The H\&E staining showed small foci of myocytolysis accompanied by small monocytic infiltrates mainly consisting of macrophages and some lymphocytes. These were only observed in the 50-wk-old rats. Therefore, we quantified the frequency of inflammation foci, as evidenced by foci of ED-1 positive macrophages and the presence of mast cells in these 50 -wk-old rats. Figure 5 shows representative examples of the macrophage staining (red cells). Macrophages were present around sites of myocytolysis. Foci of myocytolysis were more frequently present in the subendocardium and midmyocardial than subepicardial and to a lesser extend around vessels. The focus score, which gives an indication of the number of monocytic infiltrates, was $4.6 \pm 2.1$ foci/ $10 \mathrm{~mm}^{2}$ in the Dex-group, significantly higher than $1.0 \pm 0.7$ foci $/ 10 \mathrm{~mm}^{2}$ in the Sal-group $(p<0.01)$ (Fig. 6). This difference was mainly due to the presence of more inflammation foci associated with cardiomyocytolysis in the Dex-treated rats compared with the control rats.

Mast cells were mainly present around blood vessels and not associated with inflammation foci. In the Dex-group, the number of mast cells present was $33 \pm 6$, being somewhat higher than $25 \pm 4$ in the Sal-group $(p<0.05)$.

\section{DISCUSSION}

Our study shows that neonatal Dex treatment leads to a significantly lower $\mathrm{Vw}$ at $4 \mathrm{wk}$, which is still present at $8 \mathrm{wk}$, but no longer at $50 \mathrm{wk}$. These differences were only partly

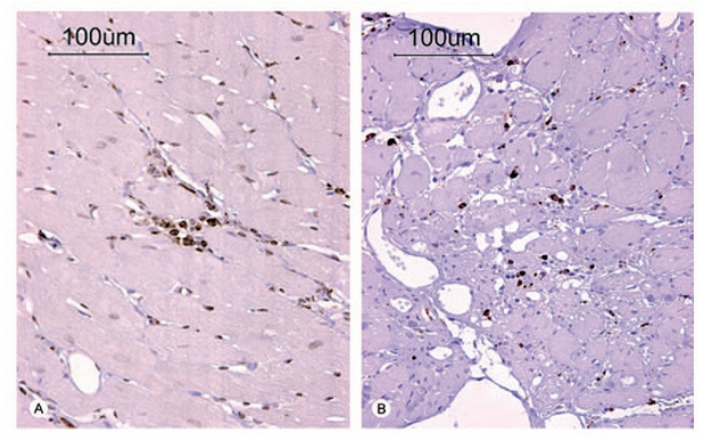

Figure 5. Macrophage staining. At $50 \mathrm{wk}$ the dexamethasone treated rats $(B)$ show more macrophage infiltrates (dark red cells) compared with saline treated rats $(A)$.

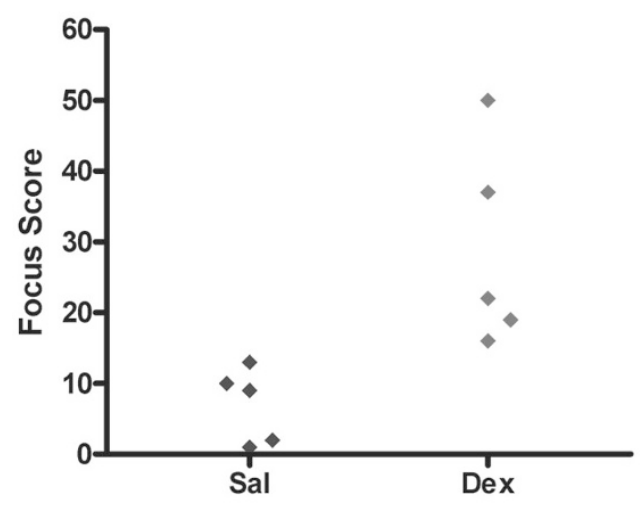

Figure 6. Macrophage focus score at $50 \mathrm{wk}$.

explained by a lower $B w$ in the Dex-treated animals, because the $\mathrm{Vw}$ : Bw ratio remained significantly reduced in the 4-wkold animals. However, at the cellular level, cardiomyocyte cell volume was increased in the Dex-treated animals, but most prominently and significantly in the 50-wk-old rats. This increased cell volume, compared with the age-matched controls, was caused primarily by an increase in cell length, whereas in the 50-wk-old animals, cardiomyocyte width was increased as well. Combining the increased cell volume with the lower $\mathrm{Vw}$ indicates a lower number of cardiomyocytes in the Dex-treated rats, which presumably is explained by a suppression of cardiomyocyte proliferation (11).

Furthermore, our data show a proportional increase in cell volume and $\mathrm{Vw}$ in the control animals (as would be expected over this age range), but the increase in cell volume is relatively accelerated in the Dex-treated animals, suggesting additional cardiomyocyte loss in the 50-wk-old rats. Moreover, in these 50-wk-old Dex-treated animals, interstitial collagen was about $60 \%$ higher and significantly more foci of macrophages were present than in controls.

To interpret the long-term effects of neonatal Dex treatment, normal myocardial development and growth should be considered. In fetal and neonatal rats, the increase in myocardial mass occurs mainly by hyperplasia (22-24). In the transition period after birth, proliferation is replaced by hypertrophy as evidenced by an increase in the percentage of binucleated myocytes, although hyperplasia still continues during the first week of life $(23,25,26)$. This transition from 
hyperplasia to hypertrophy during the early postnatal period is further influenced by nutritional, hemodynamic, and humoral factors. The increasing mechanical load (27), the perinatal increase in plasma catecholamine (28), triiodothyronine concentrations (29), and endogenous GC-production (30) accelerate the conversion from hyperplasia to hypertrophic growth. Although several studies suggest that during adult life a significant fraction of myocytes retain the ability to divide $(31,32)$, there is a physiologic decrease in the total number of cardiomyocytes in the ageing heart (33). Neonatal Dex treatment is expected to affect myocardial development through the negative effect on mitosis still occurring in the first week of life. Indeed perinatal administration of cortisol in fetal lambs was shown to inhibit myocyte hyperplasia and to stimulate the hypertrophic myocardial growth pattern, which normally starts postnatally (34). Therefore, Dex may interfere not only with the number of cardiomyocytes retaining the ability to divide but also with the total number of cardiomyocytes. In a recent study, in the newly born rat pup, we showed that the physiologic ongoing cardiomyocyte proliferation during the first days of life was suppressed during neonatal Dex treatment, leading to a premature transition to hypertrophy and resulting in a reduced number of cardiomyocytes later in life (11).

The results of this study indeed confirm a lower number of cardiomyocytes in Dex-treated adult rats, whereas cell length and cell volume were increased, indicating cellular hypertrophy, apparently compensating for the lower total number of cells. The increased cardiomyocyte length in $4-8 \mathrm{wk}$ old, but not cell width may indicate an increased left ventricular volume as first compensation for loss of cardomyocytes. In the young Dex-treated rats, Vw was reduced despite this cellular hypertrophy later in life, but in the 50-wk-old animals in which also myocyte width was increased substantially, these hypertrophic processes are likely to be compensatory. Despite these compensatory mechanisms, we recently reported on the loss of systolic cardiac function in this group of 50-wk-old Dex-treated rats (15), which may indicate that cardiomyocyte hypertrophy did not fully compensate for the lower number of cardiomyocytes. Furthermore, histologic analysis of the 50wk-old Dex-treated hearts revealed more foci of inflammation associated with myocytolysis, which may indicate "wear-out" of the remaining cardiomyocytes.

The significantly higher collagen content seen in the 50wk-old Dex-treated rats compared with the Sal-treated rats in this study may be explained by several factors. First, it is known that Dex treatment leads to elevated blood pressure and chronic hypertension is accompanied with cardiac fibrosis (12). Second, the increased number of macrophages and also the presence of lymphocytes can be regarded as low grade inflammation (35) and may be associated with increased fibrosis through activation of mediators of inflammation such as TGF-beta $(35,36)$. Third, mast cells, which were elevated in the Dex-group, can stimulate collagen expression by heart fibroblasts, by releasing its fibrogenic protease chymase, which can release TGF-beta and activate angiotensin II (36). Fourth, relative ischemia possibly induced by a lower capillary density (not determined in this study) may play a role.
Cardiomyocyte hypertrophy and increased interstitial fibrosis are histologic hallmarks of cardiac failure. Macrophages have been identified as part of the inflammatory infiltrate in the failing (human) myocardium, independent of cause. Diseases in which this phenomenon has been documented include myocarditis, chronic ischemic heart disease, and idiopathic dilated cardiomyopathy $(35,37)$.

A limitation of this study may be the method used to measure cardiac dimensions. To increase confidence, we used two validated histologic slide based methods. Although the absolute numbers on cardiomyocyte dimensions were different with the CSA and volume measurements, both methods showed the same effects of Dex.

The results obtained from the present and earlier experimental studies by our group, put into question the safety of neonatal GC treatment in the human setting. One cannot simply extrapolate the reported findings in the newborn and adult rat model to the preterm infant and adult human because the growth pattern of the human myocardial growth involves continuous proliferation of myocyte nuclei from $16 \mathrm{wk}$ of gestation to about $33 \mathrm{wk}(22,38)$. Although hyperplasia continues up to the full-term gestational age hypertrophy becomes increasingly important in the last $2-3$ mo of fetal life $(22,38)$. However, Dex treatment is exclusively used in the extremely preterm baby with chronic lung disease, when cardiomyocyte hyperplasia is still active and thereby comparable with the growth pattern as established by the rat pup in the first weeks of life. Hyperplasia only decreases during advanced maturation from 33 wk of gestation onward. Myocardial tissue of term rat pups may have a different affinity to Dex than myocardial tissue of the preterm infant; however, the cardiac hypertrophy seen during Dex treatment of newborn rat pups is similar to that seen in premature infants treated with GCs $(9,10,39)$.

The above-mentioned considerations with respect to the comparison of the effects on the rat heart of neonatal Dex treatment compared with its effect on the human preterm heart, makes it plausible that the long-term effects of neonatal Dex treatment of the preterm baby mirror the long-term effects observed in the rat heart. Given the fact that since the early $90 \mathrm{~s}$ of the last century 10,000 of preterm babies in the Western world are treated with high dosages of Dex for several weeks, a mandatory cardiovascular follow-up program should be seriously considered because secondary prevention of possible Dex-induced cardiovascular disease and hypertension, often not revealing itself until adolescence and adulthood, may be necessary.

In conclusion, neonatal Dex treatment of the rat leads to permanent histopathological changes during growth and development of the heart. At all ages investigated, neonatally Dex-treated rats have smaller hearts with larger cardiomyocytes and additional cardiomyocyte loss during adulthood compared with neonatally Sal-treated rats. Although straight extrapolation of the present results in rats to humans is somewhat hazardous, we present points of evidence leading to the belief that treatment with Dex in the preterm infant with chronic lung disease cause comparable acute and long-term changes in the heart. Further follow-up of these individuals may therefore be mandatory. 


\section{REFERENCES}

1. Kinsella JP, Greenough A, Abman SH 2006 Bronchopulmonary dysplasia. Lancet 367:1421-1431

2. Watterberg KL, Demers LM, Scott SM, Murphy S 1996 Chorioamnionitis and early lung inflammation in infants in whom bronchopulmonary dysplasia develops. Pediatrics $97: 210-215$

3. Committee on Fetus and Newborn 2002 Postnatal corticosteroids to treat or prevent chronic lung disease in preterm infants. Pediatrics 109:330-338

4. Bakker JM, van Bel F, Heijnen CJ 2001 Neonatal glucocorticoids and the developing brain: short-term treatment with life-long consequences? Trends Neurosci 24:649-653

5. Kamphuis PJ, Gardoni F, Kamal A, Croiset G, Bakker JM, Cattabeni F, Gispen WH, van Bel F, Di Luca M, Wiegant VM 2003 Long-lasting effects of neonatal dexamethasone treatment on spatial learning and hippocampal synaptic plasticity: involvement of the NMDA receptor complex. FASEB J 17:911-913

6. Murphy BP, Inder TE, Huppi PS, Warfield S, Zientara GP, Kikinis R, Jolesz FA, Volpe JJ 2001 Impaired cerebral cortical gray matter growth after treatment with dexamethasone for neonatal chronic lung disease. Pediatrics 107:217-221

7. Barrington KJ 2001 The adverse neuro-developmental effects of postnatal steroids in the preterm infant: a systematic review of RCTs. BMC Pediatr 1:1

8. Israel BA, Sherman FS, Guthrie RD 1993 Hypertrophic cardiomyopathy associated with dexamethasone therapy for chronic lung disease in preterm infants. Am J Perinatol 10:307-310

9. La Mear NS, MacGilvray SS, Myers TF 1997 Dexamethasone-induced myocardial hypertrophy in neonatal rats. Biol Neonate 72:175-180

10. Werner JC, Sicard RE, Hansen TW, Solomon E, Cowett RM, Oh W 1992 Hypertrophic cardiomyopathy associated with dexamethasone therapy for bronchopulmonary dysplasia. J Pediatr 120:286-291

11. de Vries WB, Bal MP, Homoet-van der Kraak P, Kamphuis PJ, van der Leij FR, Baan J, Steendijk P, de Weger RA, van Bel F, van Oosterhout MF 2006 Suppression of physiological cardiomyocyte proliferation in the rat pup after neonatal glucocorticosteroid treatment. Basic Res Cardiol 101:36-42

12. Kamphuis PJ, de Vries WB, Bakker JM, Kavelaars A, van Dijk JE, Schipper ME, van Oosterhout MF, Croiset G, Heijnen CJ, van Bel F, Wiegant VM 2007 Reduced life expectancy in rats after neonatal dexamethasone treatment. Pediatr Res 61:72-76

13. Kamphuis PJ, Bakker JM, Broekhoven MH, Kunne C, Croiset G, Lentjes EG, Tilders FJ, van Bel F, Wiegant VM 2002 Enhanced glucocorticoid feedback inhibition of hypothalamo-pituitary-adrenal responses to stress in adult rats neonatally treated with dexamethasone. Neuroendocrinology 76:158-169

14. Bal MP, de Vries WB, van der Leij FR, van Oosterhout MF, Berger RM, Baan J, van der Wall EE, van Bel F, Steendijk P 2005 Neonatal glucocorticosteroid treatment causes systolic dysfunction and compensatory dilation in early life: studies in 4-week-old prepubertal rats. Pediatr Res 58:46-52

15. Bal MP, de Vries WB, van Oosterhout MF, Baan J, van der Wall EE, van Bel F, Steendijk P 2008 Long-term cardiovascular effects of neonatal dexamethasone treatment: hemodynamic follow-up by left ventricular pressure-volume loops in rats. J Appl Physiol 104:446-450

16. Bruel A, Nyengaard JR 2005 Design-based stereological estimation of the total number of cardiac myocytes in histological sections. Basic Res Cardiol 100:311-319

17. van Oosterhout MF, Prinzen FW, Arts T, Schreuder JJ, Vanagt WY, Cleutjens JP, Reneman RS 1998 Asynchronous electrical activation induces asymmetrical hypertrophy of the left ventricular wall. Circulation 98:588-595

18. Vliegen HW, van der Laarse A, Huysman JA, Wijnvoord EC, Mentar M, Cornelisse CJ, Eulderink F 1987 Morphometric quantification of myocyte dimensions validated in normal growing rat hearts and applied to hypertrophic human hearts. Cardiovasc Res 21:352-357
19. Gerdes AM, Moore JA, Hines JM, Kirkland PA, Bishop SP 1986 Regional differences in myocyte size in normal rat heart. Anat Rec 215:420-426

20. Natali AJ, Turner DL, Harrison SM, White E 2001 Regional effects of voluntary exercise on cell size and contraction-frequency responses in rat cardiac myocytes J Exp Biol 204:1191-1199

21. McCrossan ZA, Billeter R, White E 2004 Transmural changes in size, contractile and electrical properties of SHR left ventricular myocytes during compensated hypertrophy. Cardiovasc Res 63:283-292

22. Mayhew TM, Pharaoh A, Austin A, Fagan DG 1997 Stereological estimates of nuclear number in human ventricular cardiomyocytes before and after birth obtained using physical disectors. J Anat 191:107-115

23. Clubb FJ Jr, Bishop SP 1984 Formation of binucleated myocardial cells in the neonatal rat. An index for growth hypertrophy. Lab Invest 50:571-577

24. Loud AV, Anversa P, Giacomelli F, Wiener J 1978 Absolute morphometric study of myocardial hypertrophy in experimental hypertension. I. Determination of myocyte size. Lab Invest 38:586-596

25. Zak R 1974 Development and proliferative capacity of cardiac muscle cells. Circ Res $35: 17-26$

26. Li F, Wang X, Capasso JM, Gerdes AM 1996 Rapid transition of cardiac myocytes from hyperplasia to hypertrophy during postnatal development. J Mol Cell Cardiol 28:1737-1746

27. Baba HA, Takeda A, Schmid C, Nagano M 1996 Early proliferative changes in hearts of hypertensive Goldblatt rats: an immunohistochemical and flowcytometrical study. Basic Res Cardiol 91:275-282

28. van Bel F, Dorrepaal CA, Benders MJ, Zeeuwe PE, van de Bor M, Berger HM 1993 Changes in cerebral hemodynamics and oxygenation in the first 24 hours after birth asphyxia. Pediatrics 92:365-372

29. Breall JA, Rudolph AM, Heymann MA 1984 Role of thyroid hormone in postnatal circulatory and metabolic adjustments. J Clin Invest 73:1418-1424

30. Murphy VE, Smith R, Giles WB, Clifton VL 2006 Endocrine regulation of human fetal growth: the role of the mother, placenta, and fetus. Endocr Rev 27:141-169

31. Nadal-Ginard B, Kajstura J, Leri A, Anversa P 2003 Myocyte death, growth, and regeneration in cardiac hypertrophy and failure. Circ Res 92:139-150

32. Kajstura J, Leri A, Finato N, Di Loreto C, Beltrami CA, Anversa P 1998 Myocyte proliferation in end-stage cardiac failure in humans. Proc Natl Acad Sci USA 95:8801-8805

33. Anversa P, Palackal T, Sonnenblick EH, Olivetti G, Meggs LG, Capasso JM 1990 Myocyte cell loss and myocyte cellular hyperplasia in the hypertrophied aging rat heart. Circ Res 67:871-885

34. Rudolph AM, Roman C, Gournay V 1999 Perinatal myocardial DNA and protein changes in the lamb: effect of cortisol in the fetus. Pediatr Res 46:141-146

35. Devaux B, Scholz D, Hirche A, Klovekorn WP, Schaper J 1997 Upregulation of cell adhesion molecules and the presence of low grade inflammation in human chronic heart failure. Eur Heart J 18:470-479

36. Akgul A, Skrabal CA, Thompson LO, Loebe M, Lafuente JA, Noon GP, Youker KA 2004 Role of mast cells and their mediators in failing myocardium under mechanical ventricular support. J Heart Lung Transplant 23:709-715

37. Azzawi M, Kan SW, Hillier V, Yonan N, Hutchinson IV, Hasleton PS 2005 The distribution of cardiac macrophages in myocardial ischaemia and cardiomyopathy. Histopathology 46:314-319

38. Huttenbach Y, Ostrowski ML, Thaller D, Kim HS 2001 Cell proliferation in the growing human heart: MIB-1 immunostaining in preterm and term infants at autopsy. Cardiovasc Pathol 10:119-123

39. Muangmingsuk S, Ingram P, Gupta MP, Arcilla RA, Gupta M 2000 Dexamethasone induced cardiac hypertrophy in newborn rats is accompanied by changes in myosin heavy chain phenotype and gene transcription. Mol Cell Biochem 209:165-173 